\title{
Les Villes imaginaires de Préfète Duffaut
}

Ou les modalités de résolution du problème de chaos urbanistique haïtien

Jean-Herald Legagneur

\section{(2) OpenEdition}

\section{Journals}

Édition électronique

URL : http://journals.openedition.org/aes/338

DOI : 10.4000/aes.338

ISSN : 2258-093X

Éditeur

Laboratoire LISAA

Référence électronique

Jean-Herald Legagneur, «Les Villes imaginaires de Préfète Duffaut », Arts et Savoirs [En ligne], 5 | 2015, mis en ligne le 15 mars 2015, consulté le 19 avril 2019. URL : http://journals.openedition.org/aes/338 ; DOI : 10.4000/aes.338

Ce document a été généré automatiquement le 19 avril 2019

Centre de recherche LISAA (Littératures SAvoirs et Arts) 


\title{
Les Villes imaginaires de Préfète Duffaut
}

\author{
Ou les modalités de résolution du problème de chaos urbanistique \\ haiitien
}

Jean-Herald Legagneur

1 L'art naïf haïtien répond à une particularité que l'on retrouve dans presque toutes les autres formes d'expressions artistiques. Celle-ci repose sur le fait que lorsque les artistes qui y évoluent ne s'attachent pas à représenter la quotidienneté dans ses contours comme dans ses travers, ils s'évertuent tout simplement à s'attaquer, au moyen de l'esthétique et par l'imaginaire, à certains problèmes auxquels se confronte la société haïtienne. Préfète Duffaut, dont Villes imaginaires ${ }^{1}$ constituent le socle de l'œuvre, n'échappe pas à ce constat. Néanmoins, même s'il n'est pas en décalage avec les visées esthétiques et les préoccupations idéologiques de ses homologues haïtiens, il se distingue d'eux en focalisant sa création sur un imaginaire qui s'empare de l'urbanisme et, à la place des décideurs haïtiens, formule avec finesse ce qu'il n'est pas anodin de considérer comme un véritable projet d'aménagement ${ }^{2}$ du territoire national. Aussi l'imagerie de la ville ${ }^{3}$ se transforme-t-elle en critères spécifiques de son écriture picturale et en démarche esthétique nourrissant constamment son inspiration, stimulant abondamment sa créativité et se posant manifestement en modalités de résolution du problème de chaos urbanistique dont souffre Haïti quasi chroniquement.

2 On se demande alors en quoi consistent ces modalités et quels en sont les mécanismes. Qu'impliquent-ils du point de vue de l'«érudition imaginaire » au sens où l'entend Nathalie Piégay-Gros ${ }^{4}$, c'est-à-dire au sens de la saisie du réel par le fictionnel? Un réel qui oscille entre le géographique et l'urbanistique, l'architectural et le rural en tant que catégories scientifiques qui se sont transformées en imaginaire pictural. Il s'agit d'interroger la façon dont Duffaut parvient à appréhender ces catégories pour en faire une construction esthétique matérialisée en images inanimées, mobilisée au service de l'organisation, de la modélisation et de l'embellissement de l'espace d'existence, du vivre et de l'agir ensemble des Haïtiens. Il s'agit aussi de déterminer le sens de cette 
démarche artistique et déceler les pistes de solutions qu'elle propose à un problème dont les incidences sont déjà trop lourdes sur la survie même du peuple haïtien.

3 Nous partirons de l'idée que la saisie des procédés utilisés dans la fabrication et la formalisation des Villes imaginaires repose sur l'analyse du mode de traitement et d'organisation des différentes structures représentées. Aussi s'avérera-t-il intéressant de déterminer leur statut ainsi que leur spécificité symbolique. Pour ce faire, nous serons donc attentifs aux notions et approches de plusieurs auteurs, dont celles d'Hélène et Gilles Menegaldo 5 sur le rapport de l'art avec les imaginaires de la ville.

\section{Genèse d'une esthétique urbaine ou d'un urbanisme imaginaire}

4 La vocation de peintre de Duffaut lui aurait été merveilleusement révélée par la sainte Vierge, selon le récit réitéré par le peintre de la vision ${ }^{6}$ qu'il dit avoir reçue qui confère une dimension mystique et surnaturelle de ses débuts dans la peinture. Étudiant ce phénomène chez plusieurs peintres haïtiens, parmi lesquels Célestin Faustin, Carlo Avierl Célius affirme que :

Dans l'univers pictural d'Haïti, des récits légendaires accompagnent la mise en circulation et conditionnent la réception des œuvres de bon nombre d'artistes, d'Hector Hyppolite (1894-1948) à André Pierre (1914-2005), de Préfète Duffaut (1923-[2012]) aux artistes du mouvement Saint-Soleil apparu dans les années soixante-dix. Une même trame semble structurer ces récits, néanmoins chaque cas présente des particularités significatives dues au profil de l'artiste, à son histoire personnelle, à ses propres implications dans l'énonciation de sa légende, aux liens établis entre celle-ci et son œuvre. L'artiste serait donc un agent actif d'un dispositif qui intègre aussi bien sa création que le discours qu'il tient sur celle-ci. Ainsi l'œuvre est au centre d'un entremêlement de récits allant de ceux qui nourrissent sa conception à ceux qui accompagnent sa réception. ${ }^{7}$

Or, pour Marie José-Nadal et Gérald Bloncourt ${ }^{8}$, Duffaut est venu à l'art du pinceau en 1944. Peu après son entrée au Centre d'art ${ }^{9}$, en 1948, où l'art naïf haïtien a émergé à l'occasion d'une "incidence esthétique ${ }^{10}$, il s'est retrouvé (entre 1949-1951) parmi les réalisateurs des fresques qui décoraient les murs de la cathédrale épiscopale de la sainte Trinité. C'est dans ces circonstances que Duffaut a commencé à peindre ses motifs, avec la réalisation d'une fresque à la cathédrale citée, Jacmel (1950), dont les ingrédients forment un réseau d'images considérées comme les prémices de ses Villes imaginaires.

Parallèlement, Albert Mangonès, architecte de formation jouant le rôle de régisseur général d'importants travaux de rénovation, d'aménagement et de modernisation de Port-au-Prince ${ }^{11}$, avait déjà commencé à alerter les autorités en les sensibilisant, à travers ses écrits ${ }^{12}$, sur le développement anarchique de cette ville. Pour lui, «Une ville qui boit, mange et qui excrète ne peut aboutir qu'à une catastrophe si on la laisse à elle-même ${ }^{13}$. Les autorités ont fait la sourde oreille et la « catastrophe annoncée » a lieu le 12 janvier 2010, avec un décompte de plus de 300000 morts $^{14}$.

7 Ainsi, dans l'état où l'espace urbain haïtien se trouve actuellement, on ne peut que déplorer que l'écho des idées de Mangonès dans l'œuvre de Duffaut n'ait pas pu résonner dans les oreilles des autorités haïtiennes ni l'éclat qui leur a été donné refléter dans leurs yeux. En d'autres termes, et plus précisément encore, comment expliquer que la seule réponse que celles-ci trouvent à donner au cas de «Jalousie » que nous avons évoqué est 
de "peinturer» des maisonnettes qui, à moindre secousse, se transformeront en de véritables tombeaux pour leurs occupants, comme ce fut très largement le cas le 12 janvier 2010. En effet, il aurait suffi que ces autorités sachent regarder ou apprennent à « voir » pour qu'elles se rendent compte de la présence dans l'œuvre de Duffaut d'autant d'ingrédients impliquant des « choses à voir et à dire ${ }^{15}$ » que de motifs qui suggèrent des actions concrètes et efficaces à entreprendre. Ainsi, à bien considérer ces peintures et en prêtant attention à l'esthétique qui les sous-tend, on ne peut manquer de constater que ce qui frappe le plus chez Duffaut, c'est non seulement son sens de l'occupation et de l'organisation de l'espace, mais plus encore la façon dont il propose de le gérer. Reste donc à préciser ce que ces peintures suggèrent symboliquement et diachroniquement. Mais auparavant, il convient d'établir l'iconographie de trois d'entre elles.

\section{Structure iconographique des œuvres}

8 Nous n'avons pas pu obtenir le droit de reproduction pour les trois œuvres que nous proposons d'analyser ici. On peut cependant les observer en consultant ce lien : http:// www.galeriemonnin.com/prefeteduffaut.html ${ }^{16}$. Ces œuvres n'ont pas de titre, elles sont classées comme suit sur le site internet indiqué : images 2 of 10 / 6 of 10 et 10 of 10 . Elles ont pour cadre une baie où une mer turquoise donne l'impression de rencontrer le ciel à l'horizon.

9 La première est caractérisée par un jeu de symétrie très bien articulé entre les différents motifs. Elle peut être considérée comme une réflexion sur le rapport d'Haïti avec le Canada, la France et les États-Unis : cette représentation confronte Haïti à ces trois puissances où Port-au-Prince est représenté en plus petite proportion face à New York, que la Statue de la liberté et le drapeau américain permettent de reconnaître. À droite, c'est la Tour Eiffel, surmontée du drapeau français, qui permet d'identifier la France. C'est aussi le cas pour le Canada qui, à gauche, est identifiable grâce à son drapeau. Ces différentes puissances sont disposées de façon telle qu'elles donnent l'impression d'enclaver Port-au-Prince. Suspendu à l'espace comme un OVNI, Washington surplombe les trois autres villes dans un ciel serein entouré de sept anges. L'ensemble forme une sorte de strate où Port-au-Prince, coincé en dessous des autres villes, ne se trouve relié à elles que par des poulies. En dehors du bleu, du gris et du vert qui sont très prononcés, c'est le blanc et le jaune qui assurent les teintes de cette œuvre dont la syntaxe semble être porteuse d'un message politique évident dont seule une analyse géo-politicostratégique, prenant en compte les intérêts et l'histoire des relations de ces puissances avec Haïti, permettrait de mesurer la portée.

10 La seconde œuvre est une construction conforme à la grammaire picturale de Duffaut: elle représente des montagnes en forme de mât de cocagne en haut desquelles la Vierge est perchée. Le coloris et la topographie sont identiques à ceux du tableau précédemment étudié.

11 La troisième enfin se distingue des deux autres, d'abord par son ton plus vif et plus nuancé, donc plus coloré avec une gamme de couleurs flamboyantes; ensuite elle n'est rien de moins qu'un village prenant la forme d'un navire où les maisons sont érigées sur des pentes abruptes.

12 L'étude iconographique de ces Villes imaginaires permet de constater qu'elles obéissent à une géométrisation de l'espace indiquant comment Duffaut le conçoit et propose d'agir 
sur lui pour pouvoir changer sa topographie. Il le divise et le subdivise, le modélise sous forme de zonage. Procédant ainsi, il semble avoir fait sienne cette proposition de Tribillon qui postulait: "C'est sans doute dans cette activité de division/affectation (diviser l'espace de la ville en zones distinctes qui reçoivent des affectations différentes ${ }^{17}$ ) que l'urbanisme montre son vrai visage. $»^{18}$ En effet, Duffaut a pris soin de révéler le « vrai visage » de son « urbanisme imaginaire » au moins de deux manières.

D'abord, il distingue les zones dites « urbaines et rurales» de celles qu'on peut taxer de "réservées ", soit aux activités agricoles, soit à des sphères de végétation. Ensuite, il distribue des «sites construits »: ponts aériens géants, routes sinueuses ceinturant les pentes et les montagnes surélevées et dominant les grandes surfaces et les rues grouillantes de minuscules individus circulant dans les deux sens, villages suspendus et reliés par des tunnels, etc. Cette forme de "figuration de l'espace ", s'érige donc en des réseaux d'images qui définissent avec véhémence la grammaire picturale duffautienne, comme pour transformer ses Villes imaginaires en de véritables textes.

\section{Le geste artistique de Duffaut : une forme d'interpellation}

14 Aussi utopique que puisse paraître un tel programme iconographique, il n'a pourtant rien d'illogique dans la mesure où il place le thème de l'espace et du bâti au centre de sa préoccupation. De ce point de vue, l'on comprend fort bien que l'artiste a poussé son imagination à un point tel qu'il a créé un « univers urbain » irréel par l'invention d'une topographie fictive qui devient, selon Hélène et Giles Menegaldo, «le foyer d'un imaginaire en acte et un tissu de rapports constituant un lieu d'intelligibilité du sujet et $\mathrm{du}$ réel saisis dans la multiplicité de leurs croisements $»^{19}$. Par ce mécanisme, Duffaut réalise exactement ce que Chris Younès entend par la « rencontre entre éthique comme manière d'être et esthétique comme vérité du sentir $\aleph^{20}$. Une rencontre qui, dans la perspective de Michelle Sustrac, bannit les frontières entre "approche sensible et observation scientifique, entre savoir et sentir, entre production de connaissance et acte créatif $»^{21}$.

Cela dit, ces « croisements » qui se transforment en une sorte de convergence de l'éthique et de l'esthétique, laquelle se situe à la confluence d'une fiction picturale et d'un imaginaire urbain, ne peuvent être interprétés que comme une invitation péremptoire lancée par Duffaut à tous ses compatriotes haïtiens, et plus spécialement à l'ensemble de ses concitoyens et à l'État haïtien en particulier. Ainsi comprise, cette invitation a pour finalité de les sensibiliser à la nécessité de mieux organiser leur lieu d'être, c'est-à-dire de mettre de l'ordre dans leur façon d'habiter dans l'espace et les sommer d'imposer de la discipline dans leur manière d'exister dans le monde, car entre habiter et exister, Duffaut a bien compris qu'il n'y a pas de frontière.

16 À cet égard, nous pouvons avancer, sans exagération et sans grand risque de nous tromper, qu'à la base de ce geste artistique émerge un acte citoyen d'une portée philosophique évidente et d'une dimension éminemment humaniste, dans la mesure où il participe d'une prise de conscience par l'artiste du sens de son existence ${ }^{22}$ dans le monde. De là découle le caractère à la fois optimiste et pragmatique de l'action créatrice duffautienne. L'on pourra sans doute reprocher à cette manière de lire cet aspect de l'œuvre de Duffaut d'être trop ambitieuse - même si nous ne prétendons pas qu'elle fasse 
l'unanimité - cependant, nul ne saurait objecter sa cohérence ni mettre en doute son intelligible bien fondé. Puisqu'il en est ainsi, l'on peut se référer à Laënnec Hurbon qui attribue à cette dimension de l'œuvre de Duffaut une fonction similaire à celle que nous avons décelée, ailleurs, dans les Noces de Cana ${ }^{23}$ en ce qu'elle tend, d'après Hurbon, vers l'atténuation de la vielle dichotomie entre "barbare » et "civilisé » jetée comme une sorte d'anathème par l'Occident sur Haïti :

[...] dans la Ville imaginaire, il n'y a plus ni Barbares ni Civilisés. Voulant se donner pour le seul monde authentique, réel, le seul qui vaille la peine d'être édifié, la Ville imaginaire et, avec elle, la Vision vodou ${ }^{24}$ et la Scène du jugement dernier qui [...] ne nous livrent pas un nouvel ordre du monde inversé, mais nous renvoient plutôt à l'opposition partout opérante dans la vie sociale, culturelle et politique entre civilisé et barbare. Une opposition enracinée dans un imaginaire qui ne s'avoue plus comme tel et auquel on reste facilement aveugle ${ }^{25}$.

17 Autant conclure avec Maximilien Laroche pour affirmer que les Villes imaginaires constituent une «image, reproduction physique d'une vision, d'une perception. Objet dont la structure reproduit celle du regard intérieur du peintre $»^{26}$. Ainsi, en nous acheminant tout droit vers ces Villes imaginaires « d'où aura disparu toute trace du barbare comme du civilisé ${ }^{27}$, renchérit Hurbon, il nous apparaît tout à fait loisible et même nécessaire de nous interroger maintenant sur le sens de ce geste artistique et sur ce qu'il porte en lui comme spécificité symbolique.

\section{Portée culturelle du geste artistique}

18 Traiter de la spécificité des Villes imaginaires revient à déterminer ce qui confère à ces peintures une valeur de symbole ou un statut d'emblème esthétique et culturel. Par conséquent, il faudrait aussi considérer ce qu'elles portent en elles comme significations ayant des similarités avec d'autres facteurs de la culture haïtienne. Pour y parvenir, deux voies, qui n'en sont en réalité qu'une, s'offrent à nous. La première concerne le « réalisme merveilleux $»^{28}$ que Jacques Stéphane Alexis a théorisé dans ses romans, notamment dans Les Arbres musiciens ${ }^{29}$; la seconde se rapporte au double paradigme lié au " pays réel » et au "pays rêvé", cher à Dany Laferrière ${ }^{30}$, mais dont la paternité revient à Édouard Glissant ${ }^{31}$.

\section{Le réalisme merveilleux}

19 Le « réalisme merveilleux », que certains confondent avec le « réalisme magique ${ }^{32}$, fut utilisé par Alexis pour désigner un genre englobant la culture du peuple haïtien dans ses diverses composantes et permettant à ce peuple anciennement colonisé d'échapper à l'aliénation postcoloniale. Aussi relie-t-il réalisme aumerveilleux, deux termes antagonistes qui s'appellent pour opérer un rapprochement entre des éléments hétérogènes et des réalités incompatibles ${ }^{33}$. Cette dialectique des contraires constitue pour Alexis un moyen de décrire une situation d'« [...] imagerie dans laquelle le peuple enveloppe son expérience et reflète sa conception du monde, sa confiance en l'homme et l'explication qu'il donne aux obstacles du progrès [...] transpose naturellement ses notions de "réalité" et de "merveilleux" dans sa vision de la réalité quotidienne ${ }^{34}$.» Selon Alexis, il est tout à fait « [...] naturel que le contenu fondamental des œuvres d'art tende à atteindre l'ensemble des problèmes qui se posent à l'homme de partout ${ }^{35}$.» De là, 
l'obligation pour l'artiste de rendre possible cette association des contraires, de montrer qu'il est concerné par le « destin collectif de son peuple » et de prouver sa capacité à agir en conséquence en incorporant « au récit le merveilleux qui est le vêtement dans lequel certains peuples enferment leur sagesse et leur connaissance de la vie ${ }^{36}$ ». Maximilien Laroche insiste aussi sur ce même rapprochement pour affirmer que :

[...] le réel n'est pas le contraire du merveilleux ni ne peut en être dissocié puisqu'il n'y a de merveilleux que comme habit, vêtement du réel. Celui-ci est cette inconnue logée au cœur du réel du merveilleux, lequel est la première dans l'ordre de l'existence, de la connaissance et de la représentation. ${ }^{37}$

On voit que les deux auteurs abordent la théorie du réalisme merveilleux de la même manière en mettant l'accent sur la capacité de cette théorie à expliciter l'hétérogénéité de la culture haïtienne tissée à partir de la confluence de la triple source autochtone, africaine et occidentale. Cette dynamique inspire la création, aide à coder, encoder et décoder les contours ou les lisières des œuvres. Elle les formalise, leur donne sens et facilite leur compréhension et leur interprétation. Aussi, ces facteurs mis en œuvre dans le processus identitaire haïtien et à l'œuvre dans l'œuvre de Duffaut créent-ils une dynamique culturelle qu'on peut considérer comme une sorte de «trianité38 ${ }^{38}$ dans la mesure où, par leur médiation et leur altération, leur juxtaposition et leur fixation, ils participent à fonder cette haïtianité qui est une autre manière de dire la culture d'Haiti. Juxtaposition suppose ici un rapport dialectique, c'est-à-dire une relation d'échange et d'emprunts mutuels fait de tensions et de contestations réciproques entre ces différentes sources.

21 Voilà pourquoi Alexis pense que l'art haïtien, dans son ensemble, doit avoir un contenu social expressif qui reflète la réalité du peuple. L'œuvre de Préfète Duffaut est, dans cette optique, assez représentative de cette double réalité sociale et culturelle. Jouant à la fois sur le réel et sur l'imaginaire en mélangeant des éléments culturels tout aussi imaginaires, cette œuvre constitue une irruption ou une «intégration dynamique du merveilleux dans le réalisme ${ }^{39} »$ ou encore une sorte d'interpénétration des deux.

\section{Du « pays réel " et du " pays rêvé » ${ }^{40}$}

La représentation que Dany Laferrière a faite de ces expressions et celle que nous venons d'analyser renvoient à une même réalité : celle d'Haïti. Toutefois, si Alexis et Laroche ont brisé les frontières entre le "merveilleux » et le "réel », il semble que pour Laferrière le «pays réel » doive être distingué du " pays rêvé » puisqu'il paraît que les deux évoquent des faits diamétralement opposés. Cette décantation est faite non seulement dans Pays sans chapeau mais aussi dans L'Énigme du retour :

Quand on observe une scène de marché / chez n'importe quel peintre de rue / on n'a pas l'impression de pénétrer / dans le marché mais que c'est plutôt le marché / qui vous pénètre en vous intoxiquant / avec ces couleurs, ces odeurs et ces saveurs ${ }^{41}$ .[...] Les mêmes paysages luxuriants reviennent pour dire que l'artiste ne peint pas le pays réel mais bien le pays rêvé. ${ }^{42}$

Cette tension entre « pays réel » et " pays rêvé » se retrouve à la fin du Pays sans chapeau dans lequel un journaliste Américain dit à un peintre : «Pourquoi peignez-vous toujours [...] des gens souriants, alors qu'autour de vous, c'est la misère et la désolation? [...] Ce que je peins, répond l'artiste, c'est le pays que je rêve. $\aleph^{43} \mathrm{La}$ dichotomie est très significative dans l'œuvre romanesque de Laferrière, car elle invite à la réflexion sur ce 
qu'est réellement le cadre de vie des Haïtiens et sur ce que ce dernier devrait être idéalement. En revanche, sur le plan strictement pictural, cette dichotomie suggère, entre autres, un modèle de ce que devrait être le cadre de vie environnemental des Haïtiens qui ne cesse de se dégrader : d'où la valeur symbolique des expressions « pays rêvé » et " pays réel ». Ainsi comprises, ces expressions sont transformées en «filtres culturels » par Laferrière qui s'évertue à les imprimer, par un jeu analogique, dans l'esprit de ses concitoyens, et donc dans l'imaginaire du peuple, pour en faire un moyen de connaissance des valeurs et des grandes préoccupations nationales. De là, peut-on dire que la peinture naïve haïtienne se voit «accorder une nouvelle dignité » dans le roman qui, pour ainsi dire, s'érige en un véritable réquisitoire en faveur du changement, non seulement dans la façon de penser et de faire des Haïtiens, mais surtout dans leur façon d'habiter dans le monde.

Cette double expression "pays réel » versus " pays rêvé » - référence intertextuelle ou mise en abyme du poème d'Édouard Glissant - a pour équivalent plastique les Villes imaginaires de Duffaut. À ce titre, la correspondance de Laferrière avec Duffaut témoigne de la rencontre de deux visions artistiques autour d'une même conviction sociale : il n'y a pas d'être sans lieu d'être. Or cette réalité constitue un véritable problème pour les Haïtiens, en ce qui concerne leur capacité à organiser et à gérer leur lieu d'être. Par conséquent, comme Alexis l'a proposé, ces créateurs ont compris que leurs œuvres ne sauraient être indifférentes à ce problème. D'où la justification de notre hypothèse de départ, à savoir que l'art naïf haïtien tend toujours, et ce dans une large mesure, vers la résolution d'un problème, alors même qu'il s'exprime différemment à travers la représentation de divers sujets.

Ainsi, l'art haïtien, à travers la dimension picturale de sa forme naïve et à travers la composante scripturale de sa version romanesque, semble, à lui seul, prendre sur sa responsabilité tout le processus de construction identitaire de ce peuple. On l'a vu avec Duffaut qui a fait de sa peinture un prétexte pour poser le problème de la façon d'habiter dans le monde des Haïtiens. Il en va de même avec Alexis et Laferrière qui ont mis les conditions de vie des Haïtiens au centre de leurs préoccupations littéraires. Aussi, pour se représenter et se dire, se singulariser et se positionner, écrivains et artistes haïtiens de toutes catégories et de tout acabit convoquent-ils à leur compte toutes sortes de paradigmes avec lesquels ils construisent une "pensée de l'action » et orchestrent une " action de la pensée ». Construction et orchestration au service desquelles ils mobilisent tout un archipel de langages avec un arc-en-ciel de mots ajustés au moyen d'un fleuve de syntaxes qui se déversent dans un vaste océan bouillonnant d'images. Le tout concourt à formuler, selon l'époque et le contexte, des concepts comme haïtianité sur le plan local, négritude sur le plan transnational, créolité et créolisation au plan régional quand on ne peut se contenter tout simplement que de l'antillanité. 


\section{NOTES}

1. Préfète Duffaut a beaucoup multiplié ses Villes imaginaires. À cette date, personne ne sait exactement combien de tableaux il a peints sous ce thème ni où ils sont conservés.

2. De petits projets farfelus, bizarres et de peu d'importance ne reflétant en rien l'esprit ni la portée de l'œuvre de Duffaut sont souvent réalisés en « hommage » à cette dernière à Jalousie, un bidonville situé à l'entrée de Pétion-ville, banlieue Est de Port-au-Prince. Récemment, des maisonnettes à risques ont été « peinturées » à la Duffaut dans le but d'offrir une autre image aux touristes qui fréquentent l'hôtel Royal Oasis qui vient d'être construit à Pétion-ville. Un autre projet de l'UNESCO, en gestation depuis en 1996, et concrétisé en 2000, avait poursuivi le même but. Voir à ce sujet Évaluation du projet Les Villes: gestion des transformations sociales et de l'environnement - UNESCO. Le Projet Jalousie: "développement intégré d'un bidonville haïtien », URL : http://www.unesco.org/most/evaluationvillesjal.pdf (consulté le 26/11/2012). Dans cette même perspective, on peut voir également Arnold Antonin, Préfête Duffaut. Piété et urbanisme imaginaire, Port-au-Prince, production Centre Pétion-Bolivar, documentaire vidéo, 44 mn, 2006.

3. Prise au sens de polis, la ville désigne ici « l'espace propre à l'exercice de la politique, en tant qu'art de régler les problèmes du vivre ensemble autant par la parole que par l'action, plutôt que par la violence», Alain Cambier, Qu'est-ce qu'une ville, Paris, Vrin, coll. "Chemins Philosophiques ", 2005, p. 9. Espace public, lieu de communauté d'intérêts et catalyseur d'actions collectives, la ville est présentée par Cambier comme « le milieu où l'homme affirme son être-aumonde, au travers de la poursuite des intérêts multiples et hétérogènes qui taraudent la société civile ", Ibid., p. 83.

4. Nathalie Piégay-Gros, L'érudition imaginaire, Genève, Librairie Droz, 2009.

5. Hélène et Gilles Menegaldo, Les imaginaires de la ville: entre littérature et arts, Presses universitaires de Rennes, 2007.

6. Il ne s'est jamais lassé de conter l'histoire de la façon dont il est venu à la peinture. Voir, Arnold Antonin Préféte Duffaut. Piété et urbanisme imaginaire, 2006 et «Laure Adler s'entretient avec Préfète Duffaut, peintre haïtien », Hors-champs, France Culture, URL: http:// www.franceculture.fr/emission-hors-champs-prefete-duffaut-2012-04-19., consulté le 13/12/2012.

7. Carlo Avierl Célius, « Célestin Faustin, un peintre haïtien face au sacré » dans Histoire et missions chrétiennes, Paris, $n^{\circ} 12$, p. 93, décembre 2009; Carlo Avierl Célius, «Hector Hyppolite : ruse et subversion ", dans Haïti en marche, Haïti, Vol XXII, $\mathrm{n}^{\circ} 36$.

8. Marie-José Nadal et Gérald Bloncourt, La peinture haïtienne, Paris, Nathan, 1986, p. 110.

9. Centre artistique et culturel fondé en 1944 par Dewitt Peters qui, dans le cadre d'une coopération haïtiano-américaine à vocation éducative, était arrivé en Haïti en février 1943 pour enseigner l'anglais. Inauguré le 14 mai 1944 par le président Elie Lescot, le Centre a été reconnu d'utilité publique et subventionné par les gouvernements haïtien et américain. Jusqu'à sa destruction lors du séisme du 12 janvier 2010, il avait continué à jouer un rôle important dans la formation de jeunes peintres.

10. Carlo Avierl Célius, «La création plastique et le tournant ethnologique en Haïti », GRADHIVA, Paris, vol.1, juin 2005, p. 71-94, p. 75.

11. À l'occasion de la préparation à la commémoration du deux-centième anniversaire de la ville de Port-au-Prince.

12. Notons que les articles écrits par Mangonès sur ce sujet ont été réunis dans un ouvrage publié en 2001. Voir, Albert Mangonès, En toute urbanité, Montréal, Éditions Mémoire, 2001. 
13. Ibid.

14. Cette ville, qui devait être un lieu de refuge pour ses habitants, s'était transformée en une sépulture, comme un "Léviathan » les expiant de leur "péché d'exister ", Cambier, op. cit., p. 44. 15. Voir Maurice Merleau-Ponty, Éloge de la philosophie, Gallimard, Coll. Folio essais, 1953, p. 45.

16. Consulté le 02 octobre 2014.

17. Jean-Paul Lacaze évoque un espace social de grande densité entendu comme lieu de mouvement et dynamisation de l'interaction, ce que l'on constate dans les peintures analysées. Voir Jean-Paul Lacaze, Les méthodes de l'urbanisme, Paris, PuF, 1990, p. 4.

18. Jean-François Tribillon, L'urbanisme, Paris, La Découverte, 2009, p. 11.

19. Hélène et Gilles Menegaldo, op. cit., p. 18.

20. Chris Younès, Art et philosophie, ville et architecture, Paris, La Découverte, 2003, p.11.

21. Michelle Sustrac, «De la ville sensible au sens de la ville » dans Hélène et Gilles Menegaldo, Les Iimaginaires de la ville..., op. cit., p. 335.

22. Nous nous inspirons ici de la proposition que Sartre a faite à propos du rôle sujet et du sens de son action dans la définition de sa propre personne dans Jean-Paul Sartre, L'Existentialisme est un humanisme, Paris, Gallimard, coll. « Folio essais », 1946.

23. Voir Jean Herald Legagneur, "Culture européenne et construction identitaire en Haïti : filiation historique et différence anthropologique des pratiques et des représentations ", MSH, Doctorales: Histoire et imaginaire dans la construction des identités en Europe et dans le bassin méditerranéen, [en ligne], URL : http://www.msh-m.fr/editions/edition-en-ligne/doctorales/lesnumeros/histoire-et-imaginaire-dans-la/article/culture-europeenne-et-construction\# (Consulté le 28 septembre 2014).

24. La graphie du mot «vodou» ne fait pas unanimité chez les auteurs haïtiens et étrangers. Certains l'écrivent avec « au », d'autres utilisent la lettre « $\mathrm{o} »$. Ici nous conservons le vocabulaire de l'auteur de la citation.

25. Laënnec Hurbon, Le Barbare imaginaire, Paris, Cerf, 1988, p. 5.

26. Maximilien Laroche, L'image comme écho. Essais sur la culture et la littérature haïtiennes, Québec, Nouvelle optique, 1978, p. 236.

27. Laënnec Hurbon, op. cit. p. 17.

28. Voir, Jacques Stephen Alexis, «Prolégomènes à un manifeste du Réalisme merveilleux des Haïtiens ", no spécial $1^{\mathrm{er}}$ Congrès international des écrivains et artistes noirs, Présence Africaine, octobre 1956, p. 245-271 et Jacques Stephen Alexis, " Où va le roman? », Présence Africaine, n¹3, avril /mai 1957, p. 81-101, un autre article traitant du même thème. Notons qu'Alejo Carpentier a fait usage de Real maravilloso dans El reino de este mundo (1949). Une cohabitation harmonieuse est possible entre le réel et le merveilleux, bien qu'ils soient apparemment opposés. Dans cette perspective, Jean-Louis Joubert a fait remarquer que Jacques Roumain et Jacques Stephen Alexis ont exploré dans leurs romans les voies du "réalisme merveilleux ", version haïtienne du «réalisme magique » des écrivains latino-américains de langue espagnole : alliance baroque du mythe et du concret, goût des images vives et d'une écriture virtuose, tropicale, travail sur une langue européenne naturalisée américaine. Cette tendance se prolonge dans les romans de Francis-Joachim Roy (Les Chiens, 1961), de René Depestre (Le Mât de cocagne, 1979 ; Hadriana dans tous mes rêves, 1988), de Jean Metellus, qui développe un cycle autour du lieu magique de sa ville natale, Jacmel (Jacmel au crépuscule, 1981.) Voir à ce propos Jean-Louis Joubert, "Caraïbes littératures ", dans Encyclopédie Universalis, URL: https://nomade.univ-tlse2.fr/http/ www.universalis-edu.com/encyclopedie/caraibes-litteratures (consulté le 29 janvier 2013).

29. Jacques Stephen Alexis, Les Arbres musiciens, Paris, Gallimard, 1957. Rappelons que L'Espace d'un cillement et Romancero aux étoiles, publiés respectivement en 1950 et 1960, se situent dans cette même lignée.

30. Dany Laferrière, Pays sans chapeau, Monaco, Le Rocher, coll. « Motifs », 2007[1999].

31. Édouard Glissant, Pays rêvé, pays réel, Paris, Gallimard, 2000. 
32. Titre d'un ouvrage de Frantz Roh (1925) où est présenté un bilan de la réalité allemande et européenne de l'art après la décadence de l'Impressionnisme et de l'Expressionnisme.

33. Jacques Rancière, Le Destin des images, Paris, La Fabrique, 2003, p. 66.

34. Précisons que cet extrait n'est pas tiré du texte de communication évoqué plus haut, mais de l'article intitulé : «Où va le roman » également évoqué plus haut. Il est cité par Zilá Bernd dans «Réalisme merveilleux», URL : http://www.flsh.unilim.fr/ditl/Fahey/ RALISMEMERVEILLEUX_n.html (consulté le 26 janvier 2013).

35. Jacques Stephen Alexis, " Prolégomènes à un manifeste ... ", op. cit. p. 247.

36. Zilá Bernd, op. cit.

37. Maximilien Laroche, Contribution à l'étude du réalisme merveilleux, Québec, GRELCA/Un. Laval, 1987, p. 125-126.

38. C'est sur le sens englobant que prend la notion des "Humanités» chez la philosophe américaine Martha Nussbaum que nous nous basons pour forger ce concept de «trianité » en combinant la racine latine "tri» (trois) et les trois dernières syllabes ( anité») des «Humanités» pour expliquer la dynamique culturelle haïtienne. Selon la philosophe, les « humanités » ne se limitent plus uniquement à l'enseignement des arts dit libéraux du trivium et du quadrivium incluant les langues grecque et latine, etc., mais elles correspondent à d'autres réalités auxquelles elle a fait références lors de son entrevue avec Julien Charnay pour le magazine en ligne Philosophie. URL: http://www.philomag.com/les-idees/reinventons-leshumanites-7908 (consulté le 24 mai 2014).

39. Jacques Stephen Alexis, op. cit. p. 264.

40. Cette section s'inspire très largement et même reprend deux paragraphes d'un autre article de l'auteur. Voir en ce sens, Jean Hérald Legagneur, "L'énigme du retour de Dany Laferrière ou quand imaginaire et urgence du social se transforment en Cahier du retour au pays natal », Québec, Voix plurielles 10.2 (2013), URL: http://brock.scholarsportal.info/journals/voixplurielles/ article/view/866 (consulté le 28 septembre 2014).

41. Dany Laferrière, L'énigme du retour, Paris, Librairie Générale Française, Coll. «Le livre de Poche $", 2011$, p.122.

42. Ibid., p. 84

43. Dany Laferrière, Pays sans chapeau, op. cit., p. 276.

\section{RÉSUMÉS}

Cet article se propose de montrer comment Préfète Duffaut s'est distingué parmi les peintres naïfs haïtiens. Alors même qu'il se situe au même plan que ses homologues en matière de visées esthétiques et de préoccupations idéologiques, il semble vouloir aller plus loin qu'eux en proposant ses Villes imaginaires (socle de son écriture picturale) comme solution au problème de chaos urbanistique dont souffre Haïti. Il s'agit de déterminer les rouages de cet urbanisme imaginaire et de faire ressortir les éléments qui se trouvent aux soubassements de l'esthétique qu'il entend dégager.

\section{INDEX}

Mots-clés : Haïti, Duffaut (Préfète), ville, science, imaginaire 
AUTEUR

JEAN-HERALD LEGAGNEUR 